\title{
Analysis of Fallacies in Below-Average Chinese Debaters' Speech From Judges' Oral Feedback
}

\author{
Liqing Wang \\ Foreign Language Department, ZiJin College of Nanjing University of Science and Technology, Nanjing, China
}

\begin{abstract}
This study aims to explore and analyze the common characteristics of fallacies made by below-average Chinese debaters and identify the criteria used by judges in evaluating those fallacies that determine the ranking of debaters. The 20 pieces of oral feedback from judges on the total fallacies made by five teams of below-average debaters from one tournament of the FLTRP CUP debate competition were selected. A direct link to the cause of faulty reasoning in the group of lower ranked teams was found between relevance-related and structural-related fallacies. Additional contributory fallacies which prevented debaters from winning if not necessarily losing were those related to acceptability and refutation, finally, the most prevalent fallacy was found to be that related to sufficiency which occurred in varying degrees and at different levels of the team.
\end{abstract}

Index Terms — fallacies, oral feedback, below-average debaters

\section{INTRODUCTION}

Chinese 2nd language learners, who are unable to use appropriate evaluation criteria to think consciously and make reasonable judgments (Paul \& elder, 2006) are generally described as lacking the skills of critical thinking, a phenomenon that teachers and researchers have spared no efforts to tackle. Arguably one of the most fundamental skills, this ability underlies success in academia and professional careers (Harrel, 2011). To master this ability, students need to know what makes a good argument and how to construct a good argument.

This situation has engendered the growth of such courses as debate at university and is the reason for the increasing popularity of the British Parliament (BP) debate in China. Indeed, to some extent, the Covid-19 virus outbreak in 2020 also played a role in it. Since debaters were unable to take part in offline tournaments, online contests became a frequent occurrence. This situation resulted in a reduction in the cost of hosting and attending such events, thereby affording participants more opportunities of participating. In addition, the relatively easy access to such events has enabled many young adjudicators with excellent credentials in the field to organize such online events, adding much diversity and innovation to the debate itself.

Accordingly, research on the cultivation of critical thinking ability has become the top priority of English teaching and scientific research. However, the current research focuses on the rules, strategies, and importance of debate, which makes little contribution to our understanding of the characteristics of debaters' critical thinking skills and how to improve these. Teachers always take it for granted that as long as they emphasize the skills needed, students will inevitably follow on with the process of analysis, reasoning, and evaluation, thereby improving their abilities in these areas through the practice of debate. However, it is often the case that after a whole semester's classroom instruction and practice, debaters were still found to be struggling with many problems such as providing adequate justifications to their claims and generating counterarguments to rebut the opposite side. Below-average debaters, in particular, find it very difficult to break the preliminary rounds, with their speaker points below the average of 75 points, given at tournaments. An evaluation of contestants' speeches showed these below-average participants exhibited fallacies of various kinds in evidence or reasoning, thus lowering the logical quality of arguments. How then, should one evaluate these fallacies? Which fallacy proved to be the crucial factor preventing contestants from being able to construct sound arguments? Fortunately, the very availability of an increased number of tournaments provides us with oral feedback from judges who, as experienced debaters, are much better qualified to evaluate participants' fallacies than teachers who have little experience of formal debate themselves. It is undeniable that relevant feedback from such debates has had a significant pedagogical impact in informing and enlightening teachers on the actual problems and pitfalls encountered by these below-average debaters. Careful analysis of this feedback enables teachers to tailor their instruction in the classroom to the areas pinpointed as being deficient.

\section{A General Review Of Fallacies In Argumentation}

\section{A. Fallacies in Argumentation}

Whether a team can win a tournament is determined by the quality of arguments. An argument is a claim supported by other claims (Toulmin,1958), but it may not be a very good one. Good arguments, even with flaws can still be persuasive but some fallacies can only lead arguments to go wrong (Facione, 2011). Therefore, recognizing and 
evaluating fallacies are essential. This thesis takes T.Edward Damer's theory of fallacy for the reason that his model identifies five criteria for good arguments and fallacies are defined as being any violation of those criteria. Since this thesis is concerned with pedagogical implications to good argumentation, what matters is not how many fallacies debaters make but what particular fallacies are frequently made and what particular fallacies lead to poor quality of the argument. Besides, comparing with Johnson and Blair's model (Trapp, 2016), Damer's five categories of fallacies, rather than three, are clearer, and more specific to help to recognize good arguments, though the two models are both based on criteria for logical assessment of arguments.

A good argument meets five logical criteria: (1) the structural demands of a well-formed argument, (2) the relevance of the argument's premises, (3) the acceptability of the argument's premises, (4) the sufficiency of the premises to support the conclusion of the argument and (5) the effectiveness of the arguments rebuttal to the strongest criticisms against the argument or the position it supports (Damer, 2008, P.2).

Accordingly, a fallacy is a violation of one or more of the five criteria of a good argument. Specifically, (1) Begging the question fallacies, fallacies of inconsistency, and fallacies of deductive inference violate the structural criterion of a good argument for they prevent the conclusions from following either necessarily or probably from the premises; (2) Fallacies that violate the relevance criterion include fallacies of irrelevant premise and fallacies of irrelevant appeal, irrelevant to the truth or merit of conclusion; (3) Both fallacies of linguistic confusion and unwarranted assumption fallacies fail to meet the conditions of the acceptability criterion, making them unacceptable to a mature and rational person. (4) Arguments that commit causal facilities and missing evidence are not sufficiently supported by appropriate evidence and thus fail to meet the sufficiency criterion. (5) The fallacies that fail to provide an effective rebuttal or engagement to the strong case of the other side can be divided into three categories: the fallacies of counter-evidence, the ad hominem fallacies, and the fallacies of the division.

\section{B. Judge's Oral Feedback}

The role of judges in a debate is significant. The function performed by the judge includes keeping order, ranking the teams, and most importantly educating the debaters. Since judges are all former debaters who have achieved outstanding success in the field of debate, their explanation of the criteria adhered to in determining rank on a team and their advice on debating skills is of great benefit in improving debaters' understanding of argumentation and enhancing their critical thinking ability.

To a large extent, judges' feedback can be further divided into three areas: general suggestions, the reasons for the ranking, and question and answer time. Clarifying how the rankings are determined and why certain teams are preferred over others, the judges, explain how they distinguish bad arguments from good ones by pointing out the particular fallacies made by each individual or team. Moreover, the evaluative comments and feedback provided by professional debate judges were invaluable in helping debaters to better understand weaknesses in argumentation and delivery.

\section{DATA SOURCE AND ReSEARCh PROCEDURES}

The present study is conducted based on one tournament of the "FLTRP CUP" National English Debating competition jointly hosted by the Foreign Language Teaching and Research Press. Inaugurated in 1997, this competition is the only national English debating event in China. This study appertains to the competition on July 24-25, 2021 when five teams of ZiJin College entered five preliminary rounds. Since round 4 is a silent round with no oral feedback being provided, 20 instances of feedback in total, were received from judges.

TABLE1

MOTIONS FOR EACH ROUND

\begin{tabular}{|c|c|}
\hline Round & Motion \\
\hline 1 & We would cancel the summer and winter vacation in primary and secondary schools. \\
\hline 2 & We would provide a universal child-care subsidy based on the number of children per household. \\
\hline 3 & We regret the rising trend of people unwilling to post comments on controversial issues on social media. \\
\hline 5 & We regret the narrative that social science/academic research should avoid any display of emotion. \\
\hline
\end{tabular}

Common characteristics shared by these teams are that they all participated in the first five preliminary rounds but did not break or gain entry to the elimination rounds and that they were all below average debaters with their total team scores of 5-8 and average speaker points of 70-74, which were below the average of 75 points given at a tournament.

The research procedures of the study include:

1) Transcript oral feedback into written form

2) Identify and calculating fallacies made by ZiJin teams.

3) Classify and summarize major fallacy types.

4) Evaluate and analyze fallacies.

5) Discuss pedagogical implications.

Since this study aims at supplying a clear description of below-average debaters' fallacies to guide for improving teaching practices in this area, specific research questions include:

(1) What major fallacies were made by below-average debaters that contribute to poor reasoning?

(2) What criteria were used by judges to evaluate fallacies of debaters and thus determine the ranking? 


\section{ANALYSIS AND DiscUSSION}

Due to the intense nature of BP debate formats which only allow 15 minutes' preparation, fallacies tend to occur more frequently. In addition, once the draw is posted and the topic announced, all judges need to keep order, take notes and determine both the rank of the teams and each speaker's points. Therefore, they do not have time to count all the fallacies made by debaters in each round. Moreover, the purpose of this paper is not to test how many fallacies occur, but from the perspective of judges, which fallacies lead to wrong reasoning. It is for this reason that judges' feedback has been chosen for closer appraisal. The fallacies judges assessed are closely related to how the rankings are determined and why certain teams are preferred over others.

In table 2, 33 fallacies were detected in twenty debates. Although this figure is not sufficient to draw a complete picture of Chinese below-average debater's fallacies, a basic understanding of possible fallacies contributing to bad or imperfect arguments can be built. In the following analysis, fallacies mentioned by judges are collected and evaluated, and the causes of these fallacies are discussed.

\section{A. Fallacies Identification and Categorization}

According to their failure to meet the criteria of good arguments, the fallacies are categorized into five major types: 1) structural-related 2) relevance-related 3) acceptability-related 4) sufficiency-related, and 5) rebuttal-related. Meanwhile, the Fallacies are further divided into four in line with their team ranks.

TABLE 2

THE FREQUENCY AND DISTRIBUTION OF FALLACY CATEGORIES

\begin{tabular}{|l|l|l|}
\hline Categories of Fallacies & Frequency & Percentage \\
\hline Structural-related & 4 & $12 \%$ \\
\hline Relevance-related & 6 & $18 \%$ \\
\hline Acceptability-related & 5 & $15 \%$ \\
\hline Sufficiency-related & 12 & $36 \%$ \\
\hline Rebuttal- related & 6 & $18 \%$ \\
\hline Total & 33 & $100 \%$ \\
\hline
\end{tabular}

The frequency and distribution of fallacy categories in terms of team ranks

- Rank 1 Rank 2 Rank 3 Rank 4

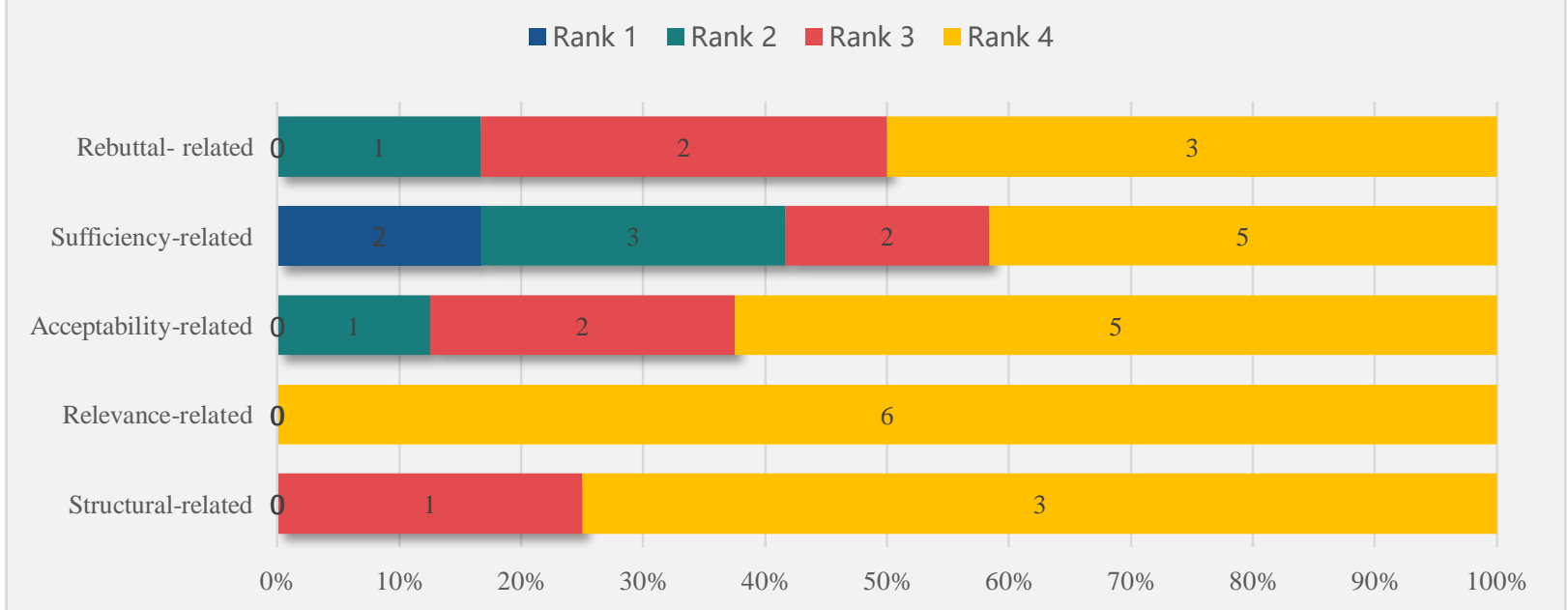

Figure 1. The frequency and distribution of fallacy categories in terms of team ranks

Generally, 33 fallacies were discussed during the judge's oral feedback time. It is evident from the data collected that sufficiency-related fallacies were the most frequently occurring of the five categories, accounting for $36 \%$. Both relevance-related and rebuttal-related fallacies were ranked second, with equal proportion $(18 \%)$ on the chart, and exceeded those of acceptability-related (15\%) and structural-related (12\%) fallacies. Moreover, the figures illustrated in general that fallacies mostly existed in outputs of lower-ranked debaters whilst higher-ranked debaters generated fewer errors.

As shown in "Fig.1", it is noteworthy that relevance-related fallacies were only attributed to the team ranked 4th. Although the third and fourth-ranked teams committed structure-related fallacies in the proportion of $25 \%$ and $75 \%$ respectively, this type of mistake didn't occur in the high-level teams. Although different levels of teams had different characteristics in generating fallacies, they all committed fallacies related to sufficiency in different proportions, ranging from $17 \%$ to $42 \%$. The following passages evaluate each fallacy type in detail and discuss how it relates to the quality of arguments.

\section{B. Fallacy Evaluation}




\section{Relevance-related Fallacy}

Based on the rule that "One who presents an argument for or against a position should set forth only reasons whose truth provides some evidence for the truth of the conclusion" (Damner, 2008, p. 92). Those who fail to do so commit a relevance-related fallacy, which does not necessarily mean the arguments presented by debaters are completely irrelevant to the motion but have low relevance or fail to attach the core idea of the topic. Of five fallacies, this category accounted for $18 \%$, but was $100 \%$ committed by teams of rank 4 , meaning that this fallacy was the most unbearable to judges and thus most detrimental to the construction of a good argument.

1) This motion is about whether researchers should display their anger, sympathy, and bias or keep calm or even cold-blooded. However, OG's case focused on researchers' interest and motivation to research. Even though this was not completely off the topic, this was not the core issue of this debate. That's why they lost this debate. (Round 5, Rank 4)

2) This is not a policy motion, so you don't need to give the policy to pre-rule out the argument of personal attack caused by controversial issues. When you did so you lost this debate because today's type of motion is "we regret", the core of which is to prove which world(factual/counterfactual) is better rather than make policy to help minimize the harm of hate speech. (Round 3, Rank 4)

The first example explains that if the conclusion that avoidance of any display of emotion in scientific research could be something harmful, the arguer needs to specifically point out why expressing emotion would not result in the inaccuracy of scientific research results. However, interest and motivation are associated with emotion but have nothing to do with their display and the objectivity of the result. Since there was no link provided to transfer the acceptability of evidence to the position, the acceptability of the conclusion was not accepted by the judge. No matter how persuasive the debater substantiated the importance of interest and concern of researcher to the scientific study, he could not win.

There are two main reasons why debaters made this mistake: One is that they were not well-informed, which couldn't be improved overnight. The other one, the lack of burden of proof (i.e. the thing you must prove to win the debate) can be corrected quickly if debaters realize that different types of motion have a different burden of proof. Like the second example provided above, the judge made it clear that the type of motion and wording determined the burden of proof. To be exact, unlike policy motion, the "we regret" motion requires both sides to weigh the advantages and disadvantages between the factual and counterfactual world according to different standards, such as "the way to make people hear".

\section{Structural-related Fallacy}

A good argument should be structurally sound, formed in a way "that the conclusion either follows necessarily or probably from its premises" (Damer, 2008, P30). Thus, the fallacies that violate the structural criterion of a good argument fail to provide us with good reasons to accept one particular conclusion. Structural-related fallacies were only committed by the teams that were ranked 3rd and 4th, with more committed by the latter (75\%) than the former $(25 \%)$. Similar to relevance-related fallacy, structural fallacies were serious ones in judges' minds.

1) As OG, to prove that students feel safer and happier by canceling the winter and summer vacation, you need to say how and why explaining the mechanism rather than simply asserting "being safer and happier is good". Without explanation, your argument doesn't stand. That's why your side lost this round. (Round 1, Rank 4)

2) Upper opposition asserted that your side of the house had an alternative and this alternative could help improve learning, but then you did not tell me the mechanism. Instead, you told me how important learning is to students. (Round 2, Rank 4)

Debaters' arguments are formed in the structure as below:

Since students can be safer and happier. (premise)

Being safer and happier is good. (implicit assumption)

Therefore, this policy (vacation cancellation) is good. (conclusion)

Since students should study well. (claim)

Therefore, training centers can help children with their learning. (conclusion)

The first example is flawed because it assumes the truth of the conclusion in its premise. This "begging-the-question fallacy" has the appearance of support like "safer and happier", but this evidence is bogus based on the implicit assumption that "being safer and happier" is equal to good. As a result, the conclusion explicitly appears as the premise, though stated in different words. The form of "since A, therefore, A." proves nothing more than "A is true because A is true". This fallacy violates the structure of a well-formed argument, a claim supported by at least one other claim, by definition. The same is true of Example 2 with the same structure. Nothing is substantially different between the premise and conclusion and thus no evidence is there to prove the credibility of the conclusion.

From oral feedback given, it is evident that those who made this type of error were awarded very low scores. Argumentation is the process whereby humans use reason to engage in critical decision-making. The emphasis on reason distinguishes argumentation from other modes of rhetoric and persuasion. When people use arguments to persuade, not only do they assert claims, but they also assert reasons why they believe their claims are plausible or probable. In a word, the debate is an activity that could not exist without argumentation (Johnson, 2010). That's why the structural-related fallacy is the most fatal error from the perspective of judges. 
To correct this type of fallacy, as one judge suggested, the debater has the burden of proof to explain 1) why we must do this, 2) how the mechanism works, 3) what proper consequences may be brought by this policy. In this motion, to be specific, a model is needed to describe how to pay the teachers, what learning activities would be offered in school, etc. Under this scenario, more details are needed to prove that students are safer and happier without vacation than when they have vacations and how "being safer and happier" can impact students.

\section{Acceptability-related Fallacy}

The fallacies discussed in this part use premises that fail to meet the acceptability criterion, meaning that evidence must be acceptable to the judge before the argument can proceed. In debate, one who presents an argument for or against the position should use reasons that are likely to be acceptable by a mature rational person. This category took up a low proportion (15\%) and except for the $1^{\text {st }}$ ranked team, teams of other levels all committed this type of fallacy.

1) I can understand the reason why people keep silent is that a lot of controversial issues are fabricated but I cannot understand why you further believed that when people stopped expressing their ideas, this was deemed social progress. (Round 3, Rank 2)

2) When the poor family is subsidized, they will use the money for gambling. This is somewhat unacceptable; I can't imagine how many families under the poverty line could do so. (Round 2, Rank 2)

3 ) It is quite weird when you said that this policy was against the interest of women of reproductive age simply because those who were reluctant to be mothers were coerced by this policy to give birth to babies. (Round 2, Rank 4)

The above three pieces of feedback are all concerned with evidence or claims unacceptable to judges. Because an individual debater might have several pieces of evidence, the acceptability of one piece of evidence might not be the main reason for the ranking allocated to him, and this fallacy sometimes mingles with other flaws, jointly reducing the quality of argumentation. For instance, the claim that a universal child-care subsidy based on the number of children per household is against the interest of women of reproductive age is both irrelevant and unacceptable. The most significant stakeholder of this policy is the family with at least one baby rather than newly married women with no babies. Even though it does pressure women of this age group, this policy also relieves the financial burden on the shoulders of those who wish to be mothers. As to the claim that when poor families are subsidized, they will use the money for gambling in the second example, a rational person would find it hard to understand why poor families who obtain that subsidy are more likely to gamble rather than satisfy their basic needs such as feeding babies with multi-nutrition.

Similarly, in example1, even though refraining from taking part in online discussion is rational, in what way can that be deemed social progress? Does it accord with common knowledge or is it supported by a recognized authority? The answer is no. Even though a lot of controversial issues are fabricated, keeping silent cannot stop this action but more importantly, saying nothing cannot help those minority groups who take advantage of controversial issues to help their voice be heard. Therefore, without other proof and examples supporting this premise, we cannot agree that "keeping silent is a social progress".

\section{Rebuttal-related Fallacy}

In debate, one side must directly refute the arguments made by the other side and show why the balance of arguments means that they have delivered enough substance to convince the judge that their arguments have importance and are backed up with specific detail and examples. Good constructive speech should include effective rebuttals to all anticipated serious criticism of the arguments. In debate, apart from the first speaker, all others are supposed to engage with strong cases from two opposite benches. The ways of arguing that fail to provide an effective rebuttal to the criticisms of ones' argument and of the position that it supports are rebuttal-related fallacies. As in the case of the acceptability-related one, rebuttal-related flaws were not seen made by the top team. These fallacies accounted for $18 \%$ of all categories. The weaker the team, the more likely it is to make such a mistake. The second and fourth-ranked teams tripled the probability of making this particular type of mistake (17\% to $50 \%$ ).

1) Opposition emphasized the critical stakeholder, the student, by specifically illustrating how this policy negatively impacted students' future. As CG, they did not engage with this strong argument. (Round 1, Rank 4)

2) When Opposition side put forward that display of emotion resulted in incorrect scientific result, Government side needs to directly compare the factual and counterfactual worlds with or without the narrative (that social science/academic research should avoid any display of emotion) in both long-term and short-time in details and highlight how emotion contribute to a more detailed and insightful conclusion. But unfortunately, your side did not succeed in doing this. Instead, you pointed out "without sympathy, all the research was meaningless" with no further explanation. (Round 5, Rank 3)

3) As a whip, it's your responsibility to refute the proposition. Though I heard you engaging with your lower house, you failed to respond to the upper whose arguments are more critical in this debate. (Round 2, Rank 2)

The first and the third example are similar in that both debaters argued in a way that omitted any reference to important evidence unfavorable to their position. We would not want to win an argument by deliberately ign oring the counter evidence under the circumstances that we know that it is damaging to our case. The leader of opposition or whip who committed this fallacy only demonstrated that they were unable to put themselves in other's shoes and since that the strong evidence was properly evaluated, no matter how cogent their arguments were, they could not win. This 
point also illustrates the fact that as the top teams in each debate, this is a fallacy one must not commit if he wants to win.

In contrast with the above two examples, the fallacy in Example 2 failed to meet the rebuttal criterion by attempting to divert attention from the actual issue. Pretending that they were engaging with the strong case from the other side, they focused critical attention on a minor point, as a result of which, their opponent's arguments remained as strong as ever.

\section{Sufficiency-related Fallacy}

The sufficiency-related fallacies, accounting for the largest proportion (36\%) were committed by all teams no matter how they ranked, fluctuating between $17 \%$ to $42 \%$. Fallacies of this type relate to evidence not being sufficient in numbers and weight to justify the acceptance of the conclusion.

1) To improve the sufficiency of DPM's speech, more details needed to be added which matched with the problem in the status quo. (Round 3, Rank 1)

2) While the leader of opposition did talk about the harm that displays of emotion can do to the scientific results, your side failed to provide a comparison between the two worlds with and with no display of emotion (Round 5, Rank 3)

3) Even though your side put forward an acceptable alternative, without further explanation, I don't know how your problem can be solved in this way. (Round1, Rank 4)

Even though sufficiency-related fallacies were made by almost all teams, they differed in forms and degrees. Specifically, some augments used too little or no evidence at all, like stated in example 3, in which missing evidence, the most crucial one, failed to bring about "alternative can solve the problem" and since the opposition was unable to solve the problem, it thus lost this debate.

Another form of this fallacy is the hasty conclusion in the second example where the debater ignored the presence of contrary evidence. She might have provided sufficient evidence to prove the harm that the display of emotion could do to the scientific results but by omitting the benefits it could bring and most importantly, without weighing up both, she could not convince the judge why the factual world was better. After all, this motion concerned "which world is better". Comparatively, the team which ranked first was much better. Even though their argument was still not fully substantiated, the key evidence was provided with a well-formed linkage to connect the claim to the conclusion.

\section{CONCLUSION}

Below average debaters' fallacies collected from one tournament "FLTRP CUP" National English Debating competition were classified and analyzed exhaustively in the present study. Some features of Chinese below-average debaters' fallacies were subsequently summarized. Meanwhile, the way judges determine the ranking by evaluating the fallacies of debaters was also highlighted based on qualitative analysis.

Fallacies existed in debaters of all ranking overall. More errors occurred in the lower levels and the error rates decreased progressively as debaters ranked higher levels. Relevance-related and structural-related fallacies were the ones that most need addressing as they were committed more frequently by Rank 4 teams, in particular, thus worthy of greater attention from teachers to improve students debating skills. Acceptability-related and rebuttal-related were types of fallacies that were shown not necessarily lead to fourth place, however, the debater who made this mistake could not win the debate. Lastly, the most frequently committed fallacies were sufficiency-related, and all teams made these mistakes to varying degrees. The lower the level, the less sufficient their arguments were.

The findings of the present study can shed light on what should be given priority in teaching practice in English classrooms in China. Attention should be shifted to bad or fallacy-laden arguments rather than instructing students on numerous specific terminologies within the field. Recognizing unsound reasoning and continuously challenging such examples in the classroom will go a long way to helping students develop their abilities to construct sound arguments which can be supported and thereby reduce the chance of fallacies occurring.

In conclusion, we may deduce several important factors that are essential for a successful debater. Firstly, having a foundation of accumulated knowledge in the subject area debated so as to be able to construct arguments to persuade the target audience. It is very important in the sense that the better-informed, the more relevant link between claim and conclusion can be achieved, but it takes time to take effect. To avoid this particular fallacy, teachers can highlight the burden of proof, that being able to back up any claims one makes is the key to the validity of his statements in the debating arena. As wording and motion types determine what burden of proof is, more exercises to analyze motion types can inspire debaters to think on the right track and avoid making relevance-related fallacies. Secondly, students should be required to reconstruct their argument into a standard format that exhibits the logical structure of an argument. With more practice, their arguments will be structurally sound. Thirdly, debating practice is not enough. Students need to reflect on their speech according to five criteria of a good argument by answering questions such as "did I give an effective rebuttal to the criticisms of my argument?', “was the premise relevant to the conclusion?", "was evidence sufficient to establish the agreement's conclusion" etc. The answer to these questions will undoubtedly improve students' critical thinking ability and help them to express their thinking more clearly. In addition, it is clear the 
development of these debating skills and knowledge are also of immense benefit and use in other areas of both students' academic and future working lives.

\section{ACKNOWLEDGMENTS}

The author would like to thank Research on philosophy and Social Sciences in Colleges and Universities in Jiang Su Province for funding this research project under grant number 2021SJA2255.

\section{REFERENCES}

[1] Damer.Edward.T.(2008). Attacking faulty reasoning: A practical Guide to Fallacy-free Arguments, Sixth Edition. Belmont: Wadsworth Publishing.

[2] Facione, P. A. (2011). Critical Thinking: What It Is and Why It Counts. Millbrae, CA: Measured Reasons and the California Academic Press.

[3] Harrell, M. (2011). Argument diagramming and critical thinking in introductory philosophy. Higher Education Research \& Development, 3, 371-385.

[4] Johnson, S. L. (2010). Win in debate. Beijing: Foreign Language Teaching and Research Press.

[5] Paul, R. \& L. Elder. (2006). Critical Thinking: Learn the Tools the Best Thinkers Use. Pearson/ Prentice Hall.

[6] Toulmin, S. (1958). The Uses of Argument. Cambridge: Cambridge University Press.

[7] Trapp, Robert. (2016). Building global relations through debate. Beijing: foreign language teaching and Research Press.

Liqing Wang was born in Nanjing, China in 1979. She received her graduate degree in linguistics from Yang Zhou University, China in 2006.

She is currently a lecturer in the Department of Foreign Languages, ZiJin College of Nanjing University of Science and Technology, Nanjing, China. Her research interests include second language acquisition and English debate. 